\title{
The impact of maternal nutrition on offspring's risk of non-communicable diseases in adulthood: a systematic review
}

\author{
Jessie Pullar ${ }^{1}$, Kremlin \\ Wickramasinghe ${ }^{1}$, \\ Alessandro R Demaio², \\ Nia Roberts ${ }^{3}$, Karla-Maria \\ Perez-Blanco ${ }^{1,}$ Katharine \\ Noonan ${ }^{1}$, Nick Townsend ${ }^{1}$ \\ ${ }^{1}$ Centre on Population Approaches for \\ NCD Prevention, Nuffield Department \\ of Population Health, University of \\ Oxford, Oxford, UK \\ ${ }^{2}$ Evidence and Programme Guidance, \\ Department of Nutrition for Health \\ and Development, World Health \\ Organisation, Geneva, Switzerland \\ ${ }^{3}$ Health Library, Nuffield Department \\ of Population Health, University of \\ Oxford, Oxford, UK
}

Background A growing body of evidence suggests the impact of maternal nutrition plays a role in determining offspring's risk of non-communicable diseases (NCDs), including heart disease (CVD), type 2 diabetes (T2DM), cancer and chronic obstructive pulmonary diseases (COPD). We conducted a systematic review to investigate this relationship.

Methods We systematically searched CINAHL, Cochrane Database of Systematic Reviews, Cochrane Register of Controlled Trials, Database of Abstracts of Reviews of Effects, MEDLINE, EMBASE, Web of Science Core Collection and Global Health for papers published before May 2016 (PROSPERO: CRD42016039244, CRD42016039247). Included studies examined the impact of maternal nutrition (diet, vitamin status and weight) on adult offspring's NCD outcomes.

Results Of 23501 identified citations, 20 met our inclusion criteria. Heterogeneity of papers required narrative synthesis. Included studies involved 1939786 participants. CVD: Four papers examined maternal exposure to famine during gestation, 3 identified a resulting increased risk of CVD in offspring. Five identified an increased risk of offspring CVD with increasing maternal weight. T2DM: Six studies investigated maternal exposure to famine during gestation; three identified an increase in offspring's T2DM risk. Three found no increased risk; two of these were in circumstances where famine states persisted beyond pregnancy. Three papers found an increased risk of T2DM in offspring with increasing maternal BMI. CANCER: Four papers investigated maternal famine exposure during pregnancy - two identified a reduced risk of cancer in male offspring, and two an increased risk in female offspring. COPD: One study found low maternal vitamin D status was associated with reduced use of asthma medication.

Conclusions While there are indications that exposure to both famine (particularly when coupled with exposure to nutritional excess after birth) and maternal overweight during pregnancy is associated with offspring's risk of CVD, T2DM and cancer, currently there is a lack of evidence to confirm this relationship. Despite the lack of conclusive evidence, these finding hold important research and policy implications for a lifecycle approach to the prevention of NCDs.

Malnutrition affects all regions of the world [1]. The double burden of malnutrition describes the co-existence of both under and over nutrition faced by individuals, households and whole populations [2]. The scale of this double burden is vast. In 2015, 462 million adults were underweight, 264 million women were affected by iron-amenable anaemia and 1.9 billion adults were overweight, with over 600 million of these obese [1]. Global action to address this double burden is es- 
sential in achieving the Sustainable Development goals and has gained political momentum through the 2014 Rome declaration on Nutrition and the UN Decade of Action on Nutrition from 2016-2025 [1,3].

Unhealthy diets are a leading risk factor for malnutrition, infant mortality and non-communicable diseases (NCDs) [3,4]. Rapid rates of globalisation and subsequent nutrition transitions has meant the burden of NCDs and over nutrition now supersedes the health burden of communicable disease and is accountable for 38 million deaths each year [3,5]. Cardiovascular disease (CVD), type 2 diabetes (T2DM), cancers and chronic obstructive respiratory diseases account for over 80\% of this NCD burden [3]. Consequently these four diseases are the focus of the World Health Organisation's (WHO) Global Action Plan for the prevention and control of NCDs (NCD GAP) [3]. The NCD GAP also focuses prevention efforts towards addressing the four common behavioural risk factors for these NCDs - tobacco use, harmful use of alcohol, physical inactivity, and unhealthy diets in childhood and adulthood [3].

While maternal nutrition has long been recognised as a determinant on fetal growth, birth weight and infant mortality [6]. A growing body of evidence suggests this influence extends well beyond childhood. In fact, maternal nutrition may play a key role in their offspring's risk of NCDs later in life. This is a profoundly important relationship, particularly in low and lower-middle income countries (LLMICs) who face the double burden of malnutrition, and 80\% of the global NCD burden [7]. Due to their impact on 'foetal programming' both maternal under- and over-nutrition have been identified as a potentially critical component of the global NCD burden $[6,8,9]$.

Currently WHO maternal nutrition targets and interventions focus on vitamin supplementation, breastfeeding and complementary feeding within the first 1000 days - from conception to two years of age [10]. Recently, the UN Secretary-General's Global Strategy for Women's and Children's Health has also recommended integrating NCDs into maternal health programmes [11]. The NCD GAP supports this recommendation, calling for a life course approach to address NCDs [3].

To ensure targeted action and effectiveness within this life course approach a strengthened evidence base is required. This systematic review aims to add to that evidence base by examining the association between maternal nutrition (prior to conception, during gestation and during lactation) and offspring's NCD outcomes. It is the first part of a two-part series. The accompanying review will examine the association between maternal nutrition prior to conception, during gestation and during lactation on the child's risk of NCDs later in life.

\section{METHODS}

Our review followed the Preferred Reporting Items for Systematic review and Meta-Analysis Protocols (PRISMA-P). The supporting PRISMA-P checklist is available in Appendix S1 in Online Supplementary Document. The current review presents a subset of the combined results from two PROSPERO registered protocols (CRD42016039244, CRD42016039247). The first outlines the methods for assessing maternal nutrition prior to and during pregnancy, while the second outlines the methods for assessing maternal nutrition during lactation. Deviation from proposed review outcomes was due to the size of magnitude of results which required division by outcome and age group. Ethics approval was not required for this review.

\section{Database and search strategies}

We conducted a comprehensive literature search for citations published before 24th May 2016. The search strategy was developed by a medical librarian in consultation with the review team. Search terms combined descriptors of pre-pregnancy, pregnancy, lactation and potential maternal nutrition factors with NCD morbidity and mortality descriptors in offspring, search terms are available in Appendix S2 in Online Supplementary Document. We conducted the search in English on the following nine databases: CINAHL, Cochrane Database of Systematic Reviews, Cochrane Register of Controlled Trials, Database of Abstracts of Reviews of Effects, MEDLINE, EMBASE, Web of Science Core Collection and Global Health. Additionally, we reviewed the reference lists of key reviews identified during screening.

\section{Inclusion criteria}

Included research assessed the impact of maternal nutrition factors measured during, or prior to pregnancy and lactation on the offspring's risk of CVD, T2DM, cancer and chronic obstructive pulmonary disease (COPD) at 18 years or older. Eligibility criteria are further outlined in Table 1. 
Table 1. Eligibility criteria

\begin{tabular}{|c|c|}
\hline MateRnal nUtRition MEASUREMENT & OfFSPRING NCD OUTCOME \\
\hline General population of mothers who: & The measurement of one of the following in offspring 18 years or older: \\
\hline $\begin{array}{l}\text { Had a nutrition indicator (including weight, weight gain, di- } \\
\text { etary intake, nutrition status) measured prior to/during preg- } \\
\text { nancy and lactation. }\end{array}$ & $\begin{array}{l}\text { Cardiovascular Disease: Diagnosed coronary heart disease (heart attack), cerebro- } \\
\text { vascular disease (stroke), peripheral vascular disease, heart failure, congenital heart } \\
\text { disease, cardiomyopathy. }\end{array}$ \\
\hline $\begin{array}{l}\text { Did not have any pre-existing chronic conditions (eg, HIV, dia- } \\
\text { betes, cardiovascular disease) }\end{array}$ & Diabetes Mellitus: Diagnosed type 2 diabetes. \\
\hline \multirow[t]{3}{*}{$\begin{array}{l}\text { Did not suffer preeclampsia, gestational diabetes or other adverse } \\
\text { conditions during pregnancy. }\end{array}$} & Cancer: Diagnosis with any form of cancer \\
\hline & Chronic respiratory diseases: Asthma, COPD, Pulmonary hypertension. \\
\hline & $\begin{array}{l}\text { Mortality outcome measures which are directly related to the above NCD's such as } \\
\text { heart attacks and stroke. }\end{array}$ \\
\hline
\end{tabular}

NCD - non-communicable disease, COPD - chronic obstructive pulmonary disease

No restrictions on publication language, location or study design were included. Studies were excluded if they did not measure a maternal nutrition factor, if they did not report on one of the predetermined outcome measures in offspring or if they were conducted on animals. Grey literature was excluded.

\section{Study selection and data extraction}

Citations from the literature search were collated in Endnote and duplicates removed. Final results were exported into Excel for eligibility screening. Using the eligibility criteria in Table $1-\mathrm{JP}, \mathrm{KP}, \mathrm{KN}$ and AF independently screened titles and abstracts. The Cohen's kappa statistic was calculated at $10 \%$ intervals (approximately every 2000 papers) to ensure consistency in screening. Once the Cohen's kappa statistic exceeded 0.75 indicating "excellent agreement", KP, KN and AF screened all remaining records. Uncertainties were brought to JP, KW and NT and disagreements resolved by group consensus. JP then reviewed all citations selected for full text review, discussing any uncertainties with KW and NT.

Following full text review, the selected studies reference lists were examined for any relevant studies. Data extraction was then conducted independently by JP on all included. Data collection involved the extraction of maternal nutrition measures, country the study was conducted in, participant characteristics, offspring NCD related outcomes (risk of NCDs), offspring age, limitations, study design, details on adjustment for confounders and quality assessment measures.

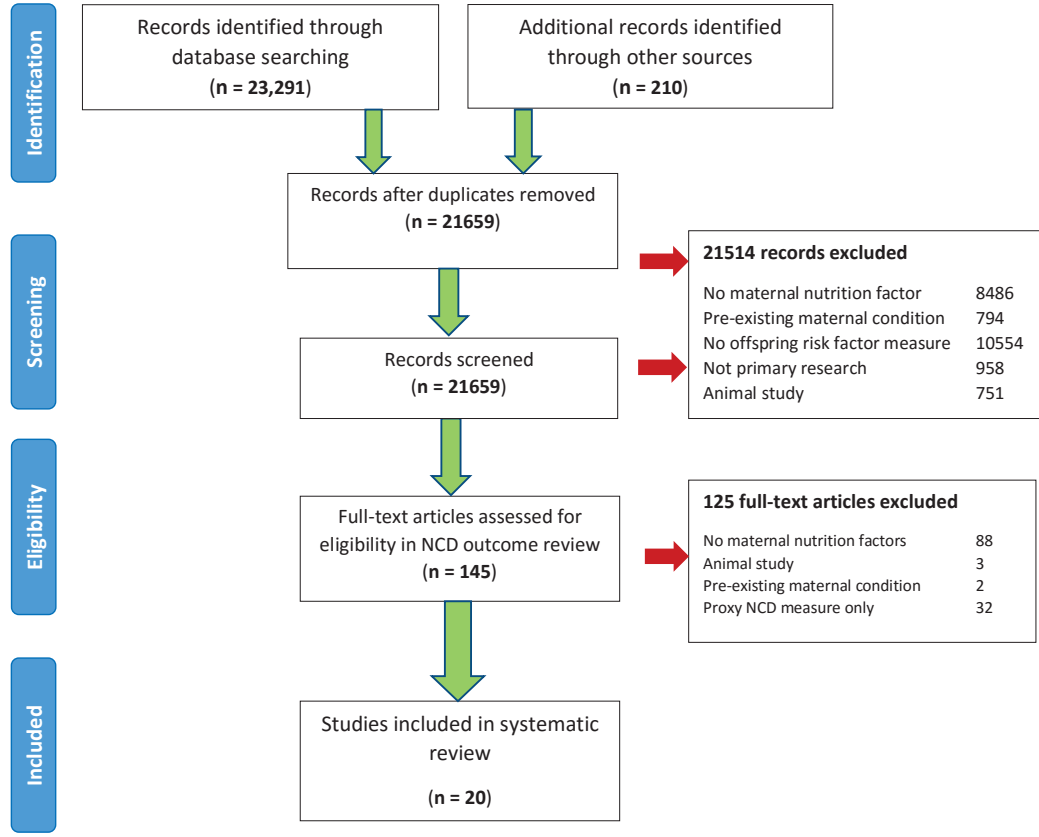

Figure 1. PRISMA flow diagram.

\section{Quality assessment}

The Newcastle-Ottawa scale (NOS) was used by JP and KW for assessing the risk of bias in cohort studies, with validated, modified versions used for cross-sectional and case-controlled studies (Appendix S3 in Online Supplementary Document) [12].

\section{Synthesis of results}

Due to the heterogeneity of the design and outcome measures in the included studies, a meta-analysis was not possible. Narrative synthesis of data was conducted by JP.

\section{RESULTS}

Our initial search returned 23501 records. After excluding 23356 records based on title and abstract screening, 145 papers were included for full-text review. Twenty studies involving 1921836 participants were included (Figure 1). 
Twelve studies were retrospective cohorts, four case-control and three cross-sectional and. Nine studies (41\%) were published before 2010. Figure 2 shows the country of origin of included studies according to WHO region. The majority (15 studies, 75\%) came from the European region, with $20 \%$ from the Netherlands. No studies from the WHO's Eastern Mediterranean region met inclusion criteria. Of the 13 countries represented, none were low income countries, three were lower-middle income countries, two were upper-middle income and the remaining eight (62\%) were high income countries.

Included studies involved nine different cohorts, four studies (20\%) involved offspring of the Dutch Famine Birth Cohort (DFBC), three examined the Helsinki Birth Cohort (HBC), and two examined the Aberdeen Birth Cohort $(\mathrm{ABC})$. Nine studies reported on the impact of maternal nutrition on CVD, nine on T2DM, six on cancer and one on COPD. The age of offspring at the time of follow up ranged from 10-69 years (with one study including participants younger than the inclusion cut off due to an inability to separate results from older subjects). Of the included studies, 10 (50\%) examined the impact of maternal exposure to famine, seven (36\%) the impact of maternal body weight, two gestational weight gain and one maternal vitamin D status, anaemia and coffee intakes impact on offspring health outcomes. No studies

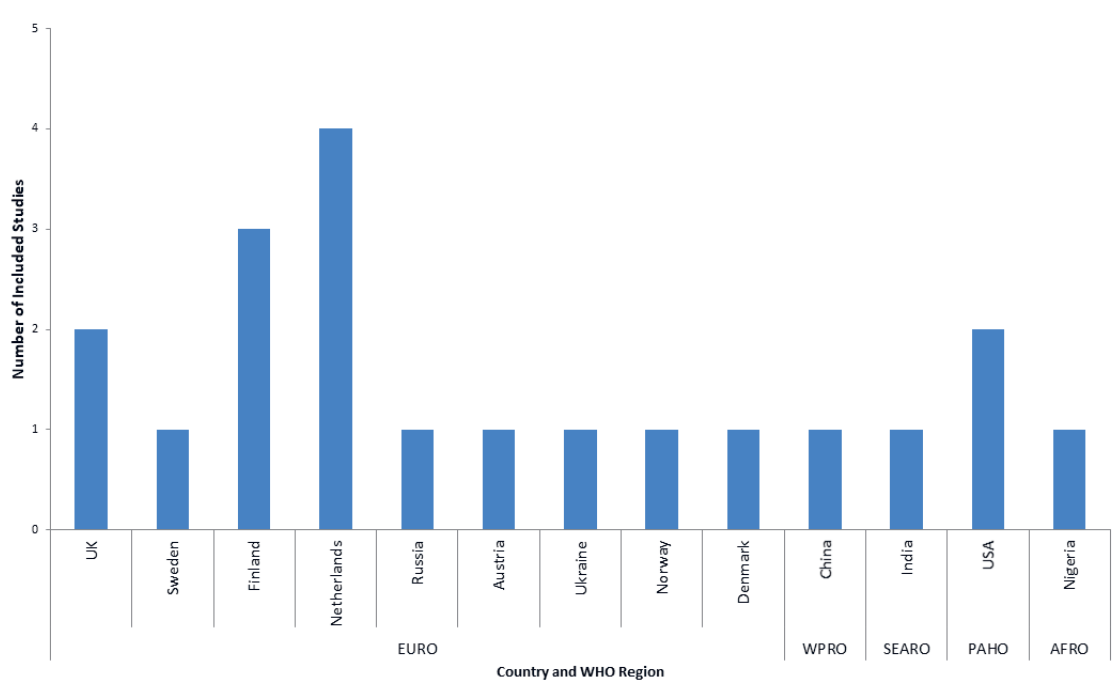

Figure 2. Countries represented by included studies according to WHO region.

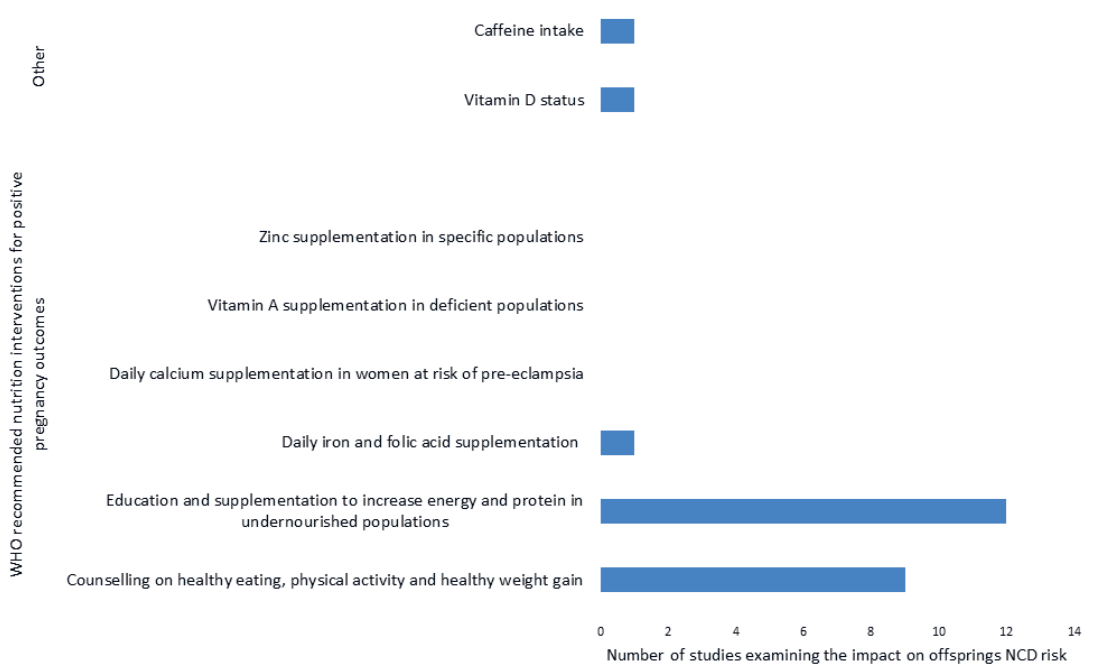

Figure 3. Number of included studies reporting outcomes related to WHO recommendations for nutrition interventions in antenatal care. evaluated the impact of maternal macronutrient intake. All studies measured these influences during gestation, with one also measuring the impact of pre-pregnancy weight. No included studies measured the impact of maternal nutrition during lactation.

When compared to current WHO recommendations for nutrition interventions during antenatal care [13], findings from included studies are consistent with existing recommendations, namely counselling on healthy diets and healthy weight/ weight gain and the prevention of undernutrition through counselling and the provision of protein and energy supplementation (Figure 3).

\section{Cardiovascular disease}

Table 2 shows nine cohort studies examined CVD outcomes in 118185 offspring. All were conducted in the European region and the majority (56\%) assessed offspring who were aged 50 years or older. Five examined the impact of maternal pre-pregnancy or pregnancy weight, four the impact of exposure to famine during pregnancy and one examined the impact of gestational weight gain. There did not appear to be an increased likelihood of identifying a significant increase in CVD outcomes due to maternal nutrition exposure based on the age of offspring when measured.

A large analysis of the ABC $(n=7134)$ found that at 50 years of age offspring of overweight and obese mothers during pregnancy had an increased risk of CVD events requiring hospital admission (15\% and 29\% respectively) [14]. The largest increase was seen for cerebrovascular disease $(61 \%$ and $54 \%$ increased 


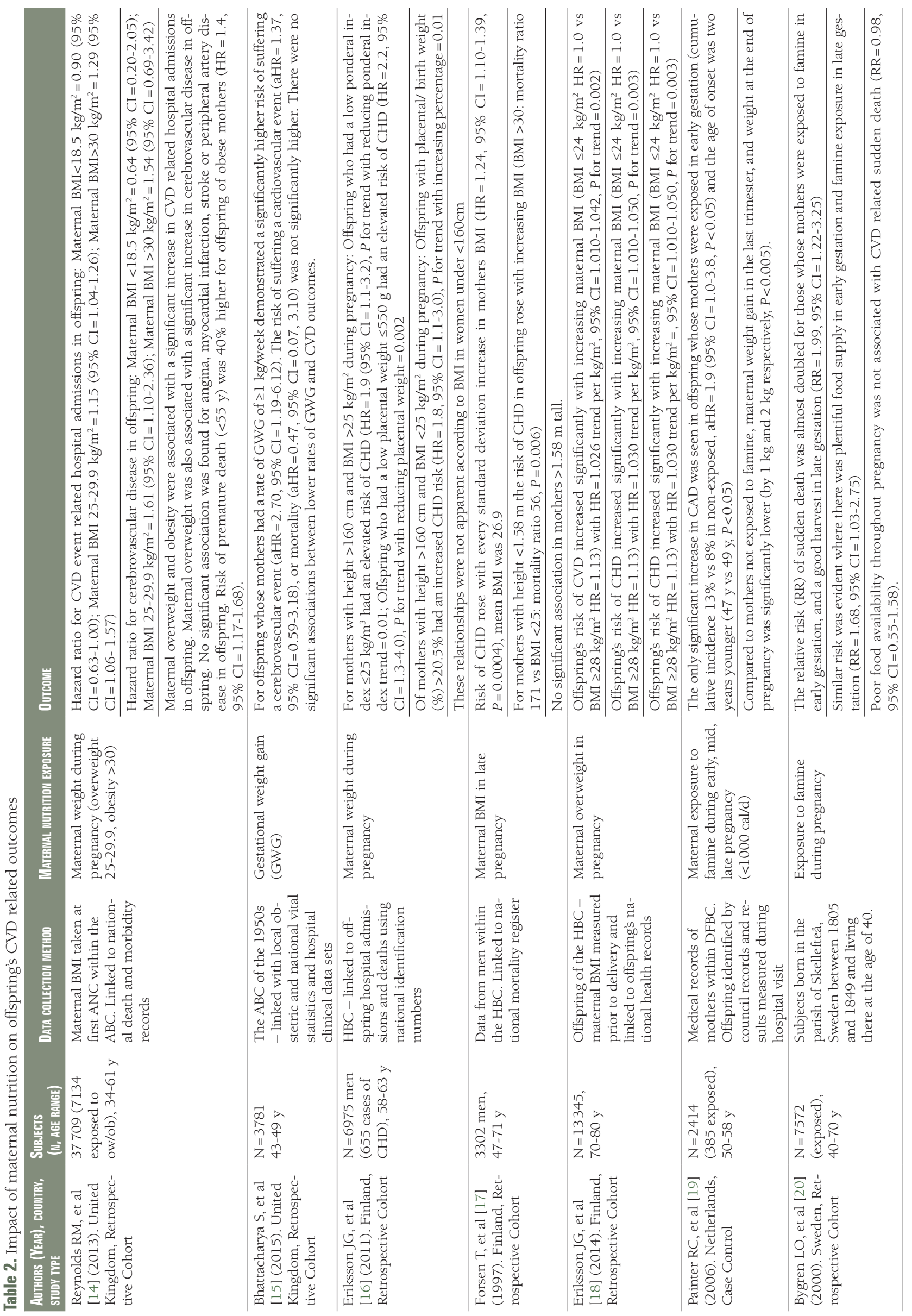


risk respectively). This is consistent with an earlier examination of the same cohort which found a 19\% increase in cerebrovascular disease risk for offspring whose mothers gained more than $1 \mathrm{~kg} /$ week [15]. No other significant associations were identified. Within this analysis it was also identified that offspring's lifestyle factors such as adult smoking, social class, BMI and diabetes status demonstrated a larger statistically significant impact on CVD outcomes [15].

An analysis specific to men born in the HBC found no association between mothers' weight and offspring's risk of coronary heart disease (CHD) [16]. When mothers were divided based on height (over or below $160 \mathrm{~cm}$ ), a significant association was found for increased risk of $\mathrm{CHD}$ in men of taller mothers. This association was mediated by mens low ponderal index $\left(<25 \mathrm{~kg} / \mathrm{m}^{3}\right.$ hazard ratio $(\mathrm{HR})=1.7,95 \%$ confidence interval $(\mathrm{CI})=1.2-2.5$ ), with $\mathrm{p}$ for trend in increased CHD risk with lower ponderal index $P=0.007$ ). Ponderal index is a measure of thinnesssimilar to BMI measures but used in children under 2 years of age, it is calculated by dividing body weight by height cubed.

When further analysed based on mothers BMI, no significant association was found for shorter mothers, though in taller mothers, offspring's CHD rose in mothers with a BMI over $26 \mathrm{~kg} / \mathrm{m}^{2}(\mathrm{HR}=1.25,95 \% \mathrm{CI}=1.10$ 1.42) per $40 \mathrm{~cm}^{2}$ decrease in placental surface area $(P$ for trend $=0.0007)$ [16]. For taller mothers, whose BMI was over $26 \mathrm{~kg} / \mathrm{m}^{2}$ offspring who had an increased risk of CHD with increasing thinness (measured by centimeter increase in the difference between length and breadth of placental surface). Low subject numbers within subgroups mean this analysis had low power. An earlier examination of the same male cohort found no significant increase in CHD related mortality for offspring of taller mothers [17]. This analysis identified an increased risk of CHD mortality for offspring of mothers who were shorter than $158 \mathrm{~cm}$ and had a BMI $>30$ in comparison to mothers with a healthy weight (mortality ratio 171 vs $56, P=0.006$ ) [17]. The final paper identified a significant, $13 \%$ increase in CVD and CHD risk for the female offspring of mothers whose BMI was over $28 \mathrm{~kg} / \mathrm{m}^{2}$ [18]. A small increase in risk was also seen for stroke in offspring of mothers whose BMI was over $28 \mathrm{~kg} / \mathrm{m}^{2}$. There was a significant trend for increased risk of CVD, CHD and stroke in offspring with each unit increase in maternal $\mathrm{kg} / \mathrm{m}^{2}, P<0.05$ [18].

Of the four studies investigating the impact of maternal exposure to famine during pregnancy, three found an increased risk of CVD in offspring [19-21]. One found maternal exposure to the Dutch famine was associated with a 5\% increased prevalence of coronary artery disease (CAD) in offspring over 50 years old, and average age of onset two years younger in those exposed in early gestation compared to non-exposed [19]. An ancient Swedish cohort also identified an increased risk of sudden death from cerebrovascular and cardiovascular disease in offspring of mothers exposed to food shortages in early or late gestation- though no significant association for those exposed to food shortages throughout pregnancy [20]. Female subjects exposed to the Dutch famine during early gestation demonstrated a hazard ratio of CVD mortality 4.6 times greater than unexposed subjects, though no significant association was found for men, or those exposed in mid to late gestation [21]. Analysis of a large, DFBC sample of male subjects found no significant association with CVD mortality [22].

\section{Type 2 diabetes mellitus}

Table 3 shows nine papers examined the impact of maternal nutrition on T2DM in offspring. Six examined the impact of exposure to famine 


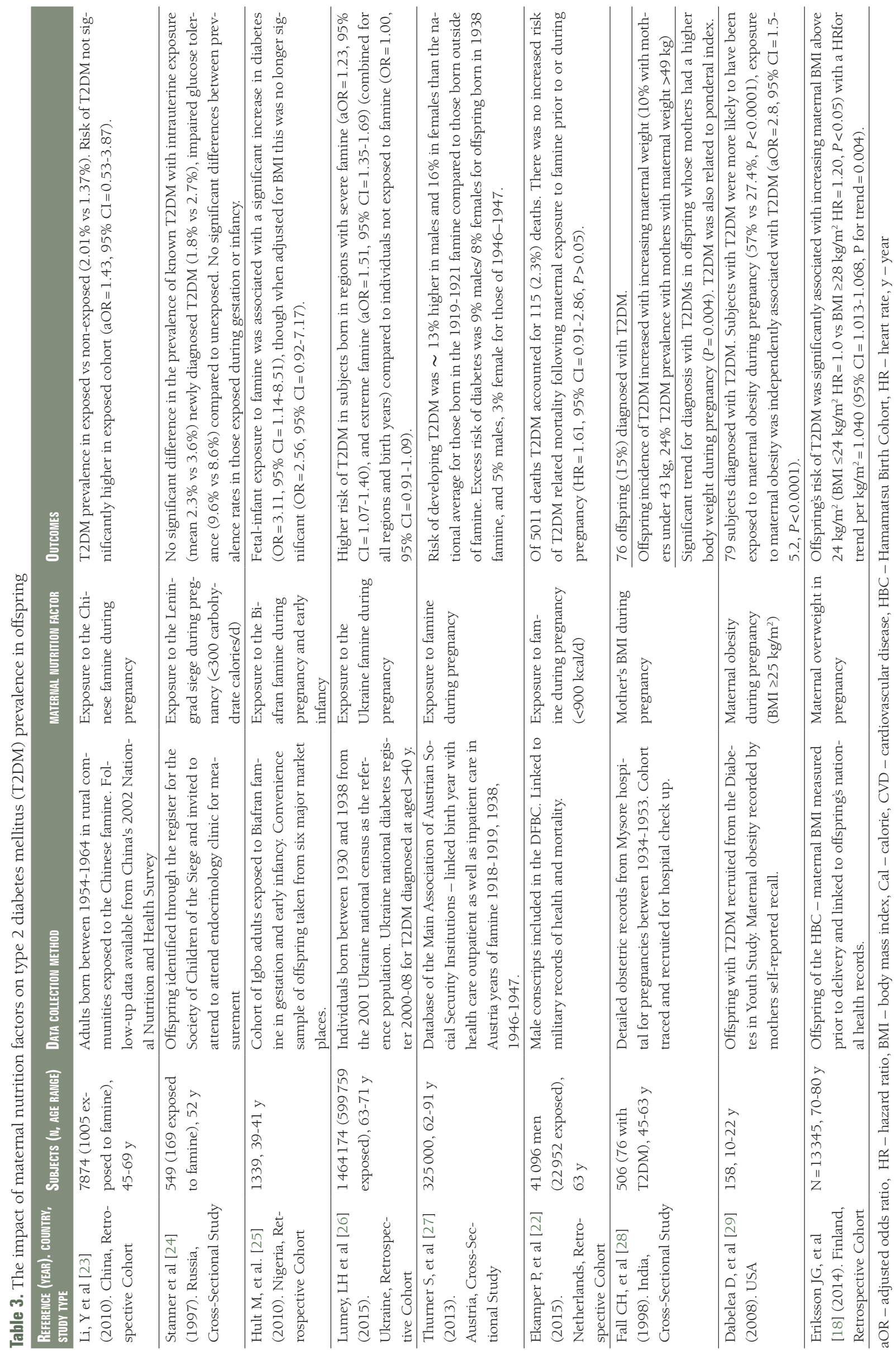


during gestation, and three investigated the impact of maternal obesity. The majority (56\%), were from the European region and measured outcomes in offspring who were 50 years of age or older (56\%). There did not appear to be a consistent association between the age of offspring's and the identification of a significant increase in T2DM due to maternal obesity or exposure to famine.

Examination of exposure to the Chinese famine during gestation on offspring's T2DM risk found no significant difference in the prevalence of T2DM between exposed and non-exposed cohorts, regardless of severity of famine exposure [23]. A small cohort of 549 subjects, examining famine exposure during pregnancy as a result of the 1941-1944 Leningrad siege also failed to find a significant impact on offspring's T2DM risk [24]. In contrast, a Nigerian cross-sectional analysis found the odds ratio of T2DM was over three times greater in offspring exposed to the Biafran famine during gestation and early infancy [25]. Though this relationship was not significant once adjusted for offspring's BMI, exposure to the famine was itself associated with increased BMI in offspring [25]. In a large cohort, adults exposed to the Ukraine famine of 1932-33 identified a dose-response relationship between severity of exposure during gestation and T2DM risk [26]. Those exposed to extreme famine during gestation showed a $47 \%$ increase in T2DM risk (adjusted odds ratio $(\mathrm{aOR})=1.47,95 \% \mathrm{CI}=1.37-1.58$ ), and a 26\% increased risk in those exposed to severe famine $(\mathrm{aOR}=1.26,95 \% \mathrm{CI}=1.14-1.39)$ in comparison to non-exposed cohorts [26]. A population analysis of all diagnosed cases of T2DM in Austria found an increased risk of T2DM in offspring born immediately after periods of famine- compared to those born outside of these periods, the significance of these relationships was not reported [27]. Analysis of a small, male DFBC subset found no significant increase in diabetes related mortality following maternal famine exposure [22].

Three studies reported on the impact of maternal obesity during pregnancy and T2DM. One small Indian cohort found a significant trend for increasing T2DM risk in offspring with increasing maternal weight during pregnancy [28]. The second American study found subjects with T2DM were significantly more likely to have mothers who were overweight during pregnancy [29]. Comparing the prevalence of maternal obesity against controls. The largest study examining this association involving the HBC found offspring's risk of T2DM was significantly associated with increasing maternal BMI with a 4\% increase in T2DM risk for every $\mathrm{kg} / \mathrm{m}^{2}$ unit increase in maternal weight ( $P$ for trend $=0.004$ ) [18]

\section{Cancer}

Table 4 shows six studies examined the impact of maternal nutrition on offspring's cancer outcomes. The majority (83\%) were conducted on European cohorts, 50\% involved offspring who were aged 50 years or older. Four studies examined the impact of maternal exposure to famine. Two studies examined this relationship in male subjects, one found males showed a reduced prevalence of testicular cancer following lower maternal weight during pregnancy due to national food shortages [30]. The other, from the DFBC, found no significant increase in cancer related mortality as a result of maternal exposure to famine -but did identify a 15\% reduction in the risk of malignant neoplasms [22]. Analysis of the DFBC cohort identified a 2 -fold increase in female offspring's cancer related mortality associated with maternal exposure to famine in early gestation ( $\mathrm{HR}=2.3,95 \% \mathrm{CI}=1.1-4.7, P=0.03)$, as well as an 8-fold increase in breast cancer mortality. No significant association was found for male offspring, or those exposed during mid or late gestation [21]. Examination of exposure to famine during gestation on offspring's breast cancer risk found an increased risk ( $\mathrm{HR}=2.6,95 \% \mathrm{CI}=0.9-7.7)$ [19].

A small cross-sectional analysis identified an increased risk of breast cancer in the offspring of women who gained 11-15 kgs during gestation $(\mathrm{OR}=1.5,95 \% \mathrm{CI}=1.1-2.0)$ [31], however there was no significant increase in risk for offspring whose mothers gained more than $15 \mathrm{~kg}$. Maternal anaemia status and coffee intake failed to show a significant relationship with offspring's breast cancer risk. Within the HBC no significant association between maternal BMI and cancer incidence, or related mortality, was found [18].

\section{Chronic obstructive pulmonary disease}

Table 5 shows only one relatively small cohort from Denmark investigated the impact of maternal nutrition on chronic obstructive respiratory disorders, specifically examining maternal vitamin D levels on asthma in offspring (126 cases of asthma). A significant reduction in asthma medication use was seen in the offspring of mothers with the lowest quartile of maternal 25(OH)D concentration, though no significant association with higher vitamin D levels, or vitamin D levels and asthma related hospitalizations [32]. 

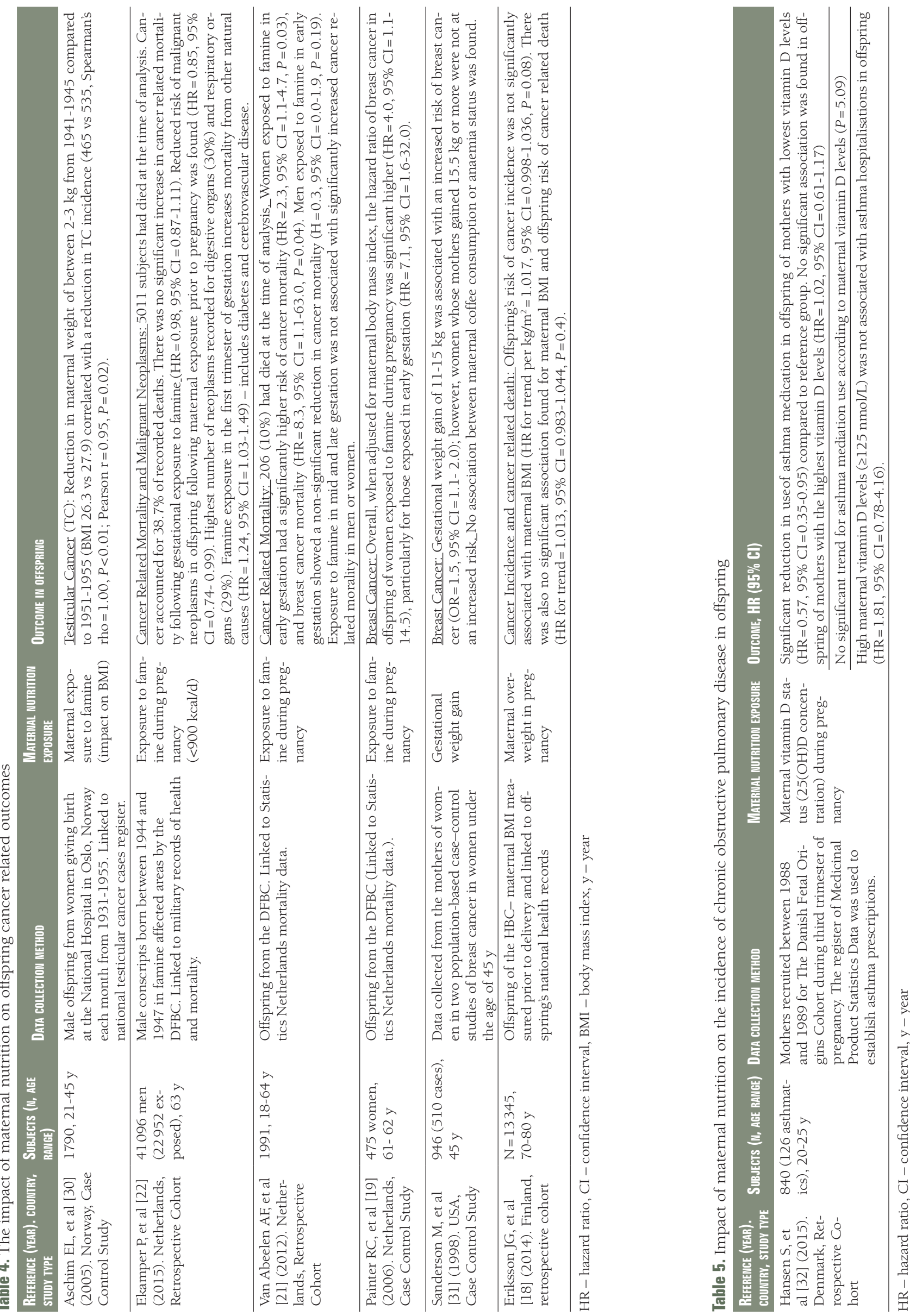


\section{DISCUSSION}

The current review demonstrated the low number of studies investigating the association between maternal nutrition and offspring's NCD risk, though available papers suggest both maternal obesity and undernourishment play an important role in offspring's risk of CVD and T2DM in adulthood. There is also some evidence that maternal undernourishment during pregnancy may increase female offspring's risk of breast cancer and cancer related mortality, while actually reducing male offspring's risk of cancer. These results are based primarily on European cohort studies.

\section{Cardiovascular disease}

All five studies investigating the impact of higher maternal BMI on CVD outcomes in offspring identified an increased risk. The largest cohorts investigating this relationship found a significant increase in CVD events between 13\%-29\% [14,18] amongst offspring of mothers with recorded BMI values over $25 \mathrm{~kg} /$ $\mathrm{m}^{2}$. Within the HBC there was also a significant trend identified for increasing risk of CVD, CHD and to a lesser extent stroke for each standard deviation point increase in BMI above $24 \mathrm{~kg} / \mathrm{m}^{2}$ [18]. Similar trends were found for CHD risk in offspring by Forsen et al [17], while an increased risk of CHD was found in male offspring of taller mothers who had a BMI over $25 \mathrm{~kg} / \mathrm{m}^{2}$, a relationship that was reliant of offspring who were also thin at birth (measured by low ponderal index) [16]. An increased risk of cerebrovascular diseases was identified in offspring of mothers who had a BMI value over $25 \mathrm{~kg} / \mathrm{m}^{2}$ [14] and mothers who gained over $1 \mathrm{~kg}$ a week during gestation [15]. Though all studies identified significant increases in risks, none indicated an increase in mortality and impacts were generally specific to cardiovascular events, coronary heart diseases and cerebrovascular disease. The largest analysis by Reynolds et al. found no relationship with the risk of angina, myocardial infarction, stroke or peripheral artery disease in offspring [14].

Maternal obesity and excessive gestational weight gain has previously been associated with a host of adverse birth outcomes, including macrosomia and childhood obesity [33,34]. A recent systematic review and meta-analysis found that once publication bias was accounted for, no protective effect was apparent for maternal obesity against low birth weight. Instead, the risk of having a very $(<1500 \mathrm{~g} R R=1.61$, $95 \% \mathrm{CI}=1.42-1.82)$, or extremely $(<1000 \mathrm{~g} \mathrm{RR}=1.31,95 \% \mathrm{CI}=1.08-1.59)$ low birth weight infant was actually increased in overweight and obese mothers [35]. This is consistent with findings of the impact of maternal weight on CVD outcomes in offspring being linked to thinness at birth which is an indicator of intrauterine growth restriction [16]. Two cohorts from the United Kingdom also identified higher rates of CHD [36] and death from ischemic heart disease [37] amongst men who had a low birth weight.

Within the four included studies, three found exposure to famine during gestation increased the risk of CVD outcomes. Each of these examined the timing of exposure to famine and found exposure during early gestation was associated with an increased risk of CAD [19], sudden death from CVD [20] and a 4-fold increase in CVD for women [21]. Within the Swedish cohort it was found that radical changes to nutrient availability during gestation, rather than chronic famine exposure throughout gestation, increased offspring's risk [20]. The largest cohort also failed to identify an increased risk of CVD related mortality as a result of famine exposure during gestation [22].

\section{Type 2 diabetes mellitus}

The six papers examining the impact of maternal exposure to famine during pregnancy on offspring's risk of T2DM found mixed results. While cohorts from Nigeria [38], Austria [27] and the Ukraine [26] found significant increases in offspring's risk, ranging from 3\% to a 3-fold increase - other large cohorts from Russia [24], China [23] and the Netherlands [22] found no increased risk. In studies which did not find an association, contextual factors may explain this null finding. For example, the DFBC subset analysis suffered from small sample sizes and limited power [22]. Within analysis of subjects exposed to the Chinese famine the timing of exposure was not well defined [23], this was also the case within the Russian cohort as it is thought that exposure to famine was not confined to pregnancy due to ongoing food shortages with Leningrad once the siege had lifted [24]. These are important considerations when results are compared to the positive findings of the larger studies from the Ukrainian and Austria which suggest a dose-response relationship with famine exposure in gestation and T2DM, particularly for those exposed in early gestation. Examination of the Ukraine famine found a dose response relationship with exposure to T2DM leading to an increased risk ranging between 26\%-47\% [26]. However, the diabetes cases within this study were taken from a unique diabetes register which maximises the likelihood of finding an association within the study. The same is true for the Austrian study by Thurner et al [27]. In 
comparison, the use of the nationally representative nutrition survey by Li et al. may be under representative of the total T2DM cases within famine affected areas [23]. Context specific factors also influence findings of a famine's impact on T2DM. For example, it should be noted that the sample population examined from the Leningrad siege was largely malnourished before the siege, and remained malnourished after the siege [24] - it is hypothesised that this meant children did not experience accelerated weight gain, thought to be a mediating factor in the link between maternal undernutrition and offspring metabolic risk factors, particularly impacting on glucose-insulin metabolism[39,40]. Supporting this theory are results from offspring of the Biafran famine in Nigeria, which indicated fetal exposure to famine was associated with higher adult BMI in offspring. While a significant increase in T2DM was not apparent once adjusted for these differences in BMI, before adjustment the risk of T2DM was three times higher in exposed infants [25]. Exposed infants also showed a significant increase in impaired glucose tolerance (aOR $=1.65,95 \% \mathrm{CI}=1.02-2.69)$ [25].

Our review identified three papers which consistently associated maternal overweight with T2DM, all indicated a significant impact with higher maternal weight linked to increased risk of T2DM in offspring. The largest of these, involving the HBC identified a 20\% increased risk of T2DM for offspring of overweight mothers (BMI $>28 \mathrm{~kg} / \mathrm{m}^{2}$ ), along with a significant trend for increasing risk with increasing maternal weight [18].

This review found some evidence to suggest an increased risk of T2DM in offspring exposed to maternal over nutrition and under-nutrition, the impact of which appears to be dependent on unhealthy lifestyle factors in offspring. Though not included within this review, studies investigating the impact of gestational diabetes on offspring's T2DM risk support the theory of trimester specific impacts as they show a trimester specific impact of a hyperglycemic intrauterine environment in mothers with gestational diabetes or type 1 diabetes, on the offspring's risk of developing T2DM [41,42]. The association is also supported by findings in some populations that a decline in T2DM prevalence correlates to reductions in low-birth weight pregnancies $[43,44]$.

This relationship between maternal nutrition status and T2DM is an important topic for future research as it could explain some of the rapidly growing prevalence rates of T2DM in LLMICs where nutrition transitions have occurred.

\section{Cancer}

Though limited, available studies investigating the impact of maternal exposure to famine before or during pregnancy suggest a 4-fold increased risk of breast cancer and 2-fold increase in cancer related mortality for female offspring - particularly for those exposed in early gestation. For men, no significant increase in cancer related mortality was identified, in line with findings of reduced occurrence of malignant neoplasms, and specifically, a reduction in testicular cancer. There was some evidence for an increased risk of breast cancer in relation to mothers GWG. This was from a small sample however and the level of GWG linked to increased risk (11-15 kg) lies within the WHO recommendations for underweight and healthy weight mothers [13], higher levels of GWG were also not associated with offspring's breast cancer risk. The larger HBC also found no association between maternal weight and offspring's risk of cancer or cancer related mortality.

Amongst 25-45 year-old Swedish men it has also been found that for each $1000 \mathrm{~g}$ increase in birthweight the risk of cancer incidence rose 17\% [45]. Findings for a reduced prevalence of testicular cancer are also consistent with an analysis of TC incidence in Scandinavian countries which shows an interruption in the increasing prevalence corresponding to men's birth during WWII [46]. This relationship also has a plausible biological explanation as higher maternal weight is associated with increased bioavailability of sex hormones including estrogen, which has been proposed as a risk factor for testicular cancer [30].

\section{Chronic obstructive pulmonary diseases}

Currently there are too few studies to form conclusions on the association between maternal nutrition and COPD. Although our search uncovered 32 papers investigating the impact of maternal nutrition factors on asthma, most measured this outcome in children aged 3-8 years and were excluded due to age restrictions. While the included paper suggests low maternal vitamin D status may reduce the risk of allergic asthma at 25 years of age, it should be noted that the study involved a relatively small sample with just 126 cases [32]. Results were however important as Denmark has been shown to have a high preva- 
lence of vitamin D deficiency as well as showing a high national prevalence of COPD [47,48]. Within the same cohort maternal obesity has also been indicated as a potential risk factor for asthma in childhood measured at seven years of age [49]. Contradictory results have been noted on the role of maternal vitamin D on asthma in offspring in previous studies [32] with a 2016 systematic review failing to identify a significant association [50].

Though no papers met the inclusion criteria of the current review, some more recent studies have indicated maternal exposure is associated with an increased risk of COPD in adult offspring [51,52].

\section{Quality assessment}

Results of assessment with the NOS (S3) found included cohort studies showed a low risk of bias for the representativeness of samples and comparators, demonstration that outcomes of interest were not present at the commencement of the study, outcome measurements and follow-up duration. The risk of bias was higher in the comparability of cohorts due to the risk of residual confounders that were not controlled for, including offspring behavioral risk factors for NCDs (diet, physical inactivity, alcohol and tobacco use), and family history of disease. The adequacy of follow-up in cohorts was also an area of potential bias with some studies reporting on only half the total cohort. Some cohorts were also limited in power due to small sample sizes within analyses. Maternal weight measurement methods and timings were mixed: some studies measured weight at only one-time point (in early or late pregnancy), and others relied on mothers' recall years after the fact.

Publication bias may be swaying results within this review. The majority of papers come from large, high quality cohorts reducing the likelihood of publication bias at a review level. At an outcome level, two papers investigating the association between maternal BMI and CVD in men reported significant findings only in small subsets of subjects, with limited power and with contrasting findings in relation to maternal height $[16,17]$. However, the association was found in a much larger cohort with low risk of bias [18].

The nature of research into maternal health means the vast majority of studies take advantage of opportunistic maternal nutrition measures, such as location and timing of birth (in reference to famines), individual maternal weight measures or routine vitamin D status checks. The review failed to identify evidence to suggest any specific foods, nutrients or maternal dietary patterns impacted on an offspring's risk of NCDs which limits the review's capacity to inform detailed nutrition advice for mothers. Animal studies have suggested more specific dietary patterns may impact on offspring's NCD risk, specifically protein deficiency, free fatty acid intake and diets high in "junk food" [41,53,54].

Availability of studies means the conclusions of this review apply primarily to European populations where cohort data are available. Currently there is a lack of evidence for examining the relationship between maternal nutrition and offspring NCD outcomes from low and lower-middle income countries where much of the global NCD burden lies. In relation to famine exposure this has important implications as the majority of included studies examine the impact of relatively short periods of famine, and opposed to extended states of food insecurity which can last generations in low income countries. A major limitation of observational studies included in our systematic review is also the risk of residual confounders influencing the results. While the majority of studies controlled for major confounders including socio-economic status, the offspring's BMI and smoking status, they could not control for family history of disease or offspring dietary and physical activity patterns. While ethical considerations limit the ability to conduct strict randomised controlled trials, interesting results have been obtained from sibling comparison studies where one sibling is born while mothers are classified as obese, and the other born after mothers have undergone gastric bypass to reduce their body weight and dietary intake. Though small $(n=111)$, this comparison found significant improvements in the siblings born after gastric bypass in relation to lower levels of severe obesity, greater insulin sensitivity and improved lipid profiles compared to those born before the gastric bypass [55]. This is an important finding as it indicates potential for prevention.

\section{Implications for the future}

While ethical considerations restrain the potential for randomised controlled trials, findings of the current review warrant further examination in future studies and reviews. These studies should focus on the measurement of more specific dietary patterns, as well as the potential of nutrition interventions to prevent undernutrition, and excessive weight gain during pregnancy and the resulting impact on birth outcomes and long term NCD risk in offspring. There is an urgent need for such research from low and lower-middle income countries. Such results could work to inform food ration programs during humanitarian crises, as well as the provision of dietary advice within antenatal care. Investigations into these fac- 
tors should align themselves to GAP NCD indicators and aim for uniformity across countries to expand the useable evidence base and strengthen the evidence case for a lifecycle approach to NCD prevention. There is also great potential to use existing databases to obtain maternal weight data with the intent to link this to offspring's NCD outcomes to add importance to this field of data.

Findings from available studies support existing WHO recommendations for nutrition interventions during antenatal care [13], namely counseling on healthy eating and healthy weight/weight gain as well as education and supplementation to increase protein and energy intake in undernourished mothers. In terms of health education, however, they suggest the impact of achieving these goals extends well beyond immediate birth outcomes and could help reduce an offspring's susceptibility to NCDs, specifically CVD, T2DM, breast cancer and cancer related mortality in the future. Findings of the impact of famine on T2DM and CVD in offspring hold important implications for many low and lower-middle income countries undergoing rapid nutritional transitions where the next generation may have been exposed to spectrums of contrast between fetal undernutrition in gestation followed by postnatal calorie excess. On the other end of the maternal nutrition spectrum, maternal obesity consistently showed an association with an increased prevalence of NCDs. Although it is difficult to control for the impacts of shared hereditary factors, environments and offspring's lifestyle factors - the consistency of these findings do suggest a critical window for impact during pregnancy. This is supported by findings of maternal BMIs impact on birthweight, and the subsequent association between birthweight and NCDs later in life. These findings hold important implications for both policy makers and practitioners, as it shows added importance, and urgency of promoting healthy weight, and weight gain during pregnancy.

\section{CONCLUSIONS}

The shortage of studies identified by this review is in itself a surprising finding considering that maternal weight, and vitamin deficiencies are commonly recorded in the maternal health protocols for many countries.

While the level of evidence is not high enough to prove causation, included studies did provide relatively consistent findings to indicate both exposure to famine, and high maternal body weight may increase an offspring's risk of CVD (particularly cerebrovascular disease) and T2DM. This relationship appears to be stronger for female offspring exposed during early gestation. In the case of famine exposure and T2DM, these diseases appear reliant upon an offspring's exposure to contrasting nutritional environments after birth. For female offspring, both exposure to famine and maternal overweight during gestation was also associated with increased risk of cancer, specifically breast cancer. For men, exposure to famine during gestation appears to be associated with reduced risk of cancer. Though there are some indications of an association, currently there are too few studies to make conclusions on the impact of maternal nutrition on adult offspring's COPD outcomes.

Acknowledgements: Authors would like to acknowledge Dr João Breda, World Health Organisation Regional Office for Europe for his inputs for the protocol and Alex Flemming, University of Oxford for his contribution to the search. Funding: Centre on Population Approaches for Non-Communicable Disease Prevention. KW and NT are funded by the British Heart Foundation (006/P\&C/CORE/2013/OXFSTATS). Review funded by the World Health Organisation (OUC13647). The World Health Organisation gratefully acknowledges the financial contribution of The Bill $\&$ Melinda Gates Foundation towards the development of systematic reviews of the evidence on the effects of nutrition interventions.

Authorship contributions: Conceptualisation: AD, KW, JP, NT. Funding acquisition: AD, KW. Performed the literature search: NR. Data Screening: JP, KP, KN, Data extraction: JP, KN, KW, NT. Analyzed the data: JP, KW, NT. Project Administration: KW, AD. Writing - original draft preparation: JP. Writing - review and editing: JP, KW, NT, AD. Provided methodological advice and critically revised the manuscript: KW, NT, AD.

Competing interests: Kremlin Wickramasinghe and João Breda are currently staff members of the World Health Organisation. The authors alone are responsible for the views expressed in this publication and they do not necessarily represent the decisions, policy or views of the World Health Organisation. The authors have completed the Unified Competing Interest form at www.icmje.org/coi_disclosure.pdf (available on request from the corresponding author) and declare no further competing interests.

Additional material

Online Supplementary Document 
1 United Nations. 71 Session of the United Nations General Assembly The UN Decade of Action on Nutrition Working together to implement the outcomes of the Second International Conference on Nutrition A side-event co-hosted by Ecuador, Italy, Japan, the Russian Federation. Rome: Food and Agricultural Organisation. Available: http://www.fao.org/ fileadmin/user_upload/lon/UN\%20Decade\%20of\%20Action\%20on\%20Nutrition\%20-\%20Concept\%20Note.pdf. Accessed: 1 November 2016.

2 Kolčić I. Double burden of malnutrition: A silent driver of double burden of disease in low- and middle-income countries. J Glob Health. 2012;2:020303. Medline:23289074 doi:10.7189/jogh.02.020303

3 World Health Organization. Global action plan for the prevention and control of noncommunicable diseases 2013-2020. Geneva: World Health Organisation. Available: http://apps.who.int/iris/bitstream/10665/94384/1/9789241506236_eng. pdf. Accessed: 22 September 2016.

4 World Health Organization. Malnutrition. Geneva: World Health Organisation. Available: http://www.who.int/newsroom/fact-sheets/detail/malnutrition. Accessed: 22 March 2017.

5 World Health Organisation. Globalization, Diets and Noncommunicable Diseases. Geneva: World Health Organisation. Available: http://apps.who.int/iris/bitstream/handle/10665/42609/9241590416.pdf?sequence=1. Accessed: 15 November 2016.

6 World Health Organisation. Good maternal Nutrition- the best start to life. Copenhagen:WHO Regional Office for Europe. Available: http://www.euro.who.int/_data/assets/pdf_file/0008/313667/Good-maternal-nutrition-The-best-startin-life.pdf?ua=1. Accessed: 10 June 2016.

7 World Health Organization. Global status report on noncommunicable diseases 2014. Geneva: World Health Organization. Available: https://www.who.int/nmh/publications/ncd-status-report-2014/en/. Accessed: 15 June 2016.

8 Snodgrass JJ, Sorensen MV. Tarskaia L a, Leonard WR. Adaptive dimensions of health research among indigenous Siberians. Am J Hum Biol. 2007;19:165-80. Medline:17286259 doi:10.1002/ajhb.20624

9 Blackmore HL, Ozanne SE. Maternal diet-induced obesity and offspring cardiovascular health. J Dev Orig Health Dis. 2013;4:338-47. Medline:24970727 doi:10.1017/S2040174412000761

10 World Health Organization. Indicators for the Global Monitoring Framework on Maternal, Infant and Young Child Nutrition. Geneva: World Health Organization. Available: http://www.who.int/nutrition/topics/indicators_monitoringframework_miycn_background.pdf?ua=1. Accessed: 10 June 2016.

11 United Nations. The global strategy for women's, children's and adolescents health (2016-2030). Geneva: World Health Organization. Available: http://www.who.int/life-course/partners/global-strategy/globalstrategyreport2016-2030-lowres. pdf. Accessed: 10 June 2016.

12 Wells GA, Shea B, O'connell D, Peterson JEA, Welch V, Losos M, et al. The Newcastle-Ottawa Scale (NOS) for assessing the quality of nonrandomised studies in meta-analyses. Otawa: The Ottawa Hospital. Available: http://www.ohri.ca/programs/clinical_epidemiology/oxford.asp. Accessed: 30 September 2016.

13 World Health Organization. WHO recommendations on antenatal care for a positive pregnancy experience. Geneva: World Health Organization. Available: https://www.who.int/reproductivehealth/publications/maternal_perinatal_health/ anc-positive-pregnancy-experience/en/. Accessed: 20 February 2017.

14 Reynolds RM, Allan KM, Raja EA, Bhattacharya S, McNeill G, Hannaford PC, et al. Maternal obesity during pregnancy and premature mortality from cardiovascular event in adult offspring: follow-up of 1323275 person years. BMJ. 2013;347:f4539. Medline:23943697 doi:10.1136/bmj.f4539

15 Bhattacharya S, McNeill G, Raja EA, Allan K, Clark H, Reynolds RM, et al. Maternal gestational weight gain and offspring's risk of cardiovascular disease and mortality. Heart. 2016;102:1456-63. Medline:27173505 doi:10.1136/heartjnl-2015-308709

16 Eriksson JG, Kajantie E, Thornburg KL, Osmond C, Barker DJP. Mothers body size and placental size predict coronary heart disease in men. Eur Heart J. 2011;32:2297-303. Medline:21632601 doi:10.1093/eurheartj/ehr147

17 Forsén T, Eriksson JG, Tuomilehto J, Teramo K, Osmond C, Barker DJP. Mother's weight in pregnancy and coronary heart disease in a cohort of Finnish men: follow up study. BMJ. 1997;315:837-40. Medline:9353502 doi:10.1136/ bmj.315.7112.837

18 Eriksson JG, Sandboge S, Salonen MK, Kajantie E, Osmond C. Long-term consequences of maternal overweight in pregnancy on offspring later health: Findings from the Helsinki Birth Cohort Study. Ann Med. 2014;46:434-8. Medline:24910160 doi:10.3109/07853890.2014.919728

19 Painter RC, de Rooij SR, Bossuyt PM, Simmers TA, Osmond C, Barker DJ, et al. Early onset of coronary artery disease after prenatal exposure to the Dutch famine. Am J Clin Nutr. 2006;84:322-7. Medline:16895878 doi:10.1093/ajcn/84.2.322

20 Bygren LO, Edvinsson S, Broström G. Change in food availability during pregnancy: Is it related to adult sudden death from cerebro- and cardiovascular disease in offspring? Am J Hum Biol. 2000;12:447-53. Medline:11534035 doi:10.1002/1520-6300(200007/08)12:4<447::AID-AJHB3>3.0.CO;2-M

21 van Abeelen AFM, Veenendaal MVE, Painter RC, de Rooij SR, Dijkgraaf MGW, Bossuyt PMM, et al. Survival effects of prenatal famine exposure. Am J Clin Nutr. 2012;95:179-83. Medline:22170371 doi:10.3945/ajcn.111.022038

22 Ekamper P, van Poppel F, Stein AD, Bijwaard GE, Lumey LH. Prenatal famine exposure and adult mortality from cancer, cardiovascular disease, and other causes through age 63 years. Am J Epidemiol. 2015;181:271-9. Medline:25632050 doi:10.1093/aje/kwu288

23 Li Y, He Y, Qi L, Jaddoe VW, Feskens EJM, Yang X, et al. Exposure to the Chinese famine in early life and the risk of hyperglycemia and type 2 diabetes in adulthood. Diabetes. 2010;59:2400-6. Medline:20622161 doi:10.2337/db10-0385 
24 Stanner SA, Bulmer K, Andres C, Lantseva OE, Borodina V, Poteen VV, et al. Does malnutrition in utero determine diabetes and coronary heart disease in adulthood? Results from the Leningrad siege study, a cross sectional study. BMJ. 1997;315:1342-8. Medline:9402775 doi:10.1136/bmj.315.7119.1342

25 Hult M, Tornhammar P, Ueda P, Chima C, Bonamy A-KE, Ozumba B, et al. Hypertension, diabetes and overweight: looming legacies of the Biafran famine. PLoS One. 2010;5:e13582. Medline:21042579 doi:10.1371/journal.pone.0013582

26 Lumey LH, Khalangot MD, Vaiserman AM. Association between type 2 diabetes and prenatal exposure to the Ukraine famine of 1932-33: a retrospective cohort study. Lancet Diabetes Endocrinol. 2015;3:787-94. Medline:26342852 doi:10.1016/S2213-8587(15)00279-X

27 Thurner S, Klimek P, Szell M, Duftschmid G, Endel G, Kautzky-Willer A, et al. Quantification of excess risk for diabetes for those born in times of hunger, in an entire population of a nation, across a century. Proc Natl Acad Sci U S A. 2013;110:4703-7. Medline:23487754 doi:10.1073/pnas.1215626110

28 Fall CH, Stein CE, Kumaran K, Cox V, Osmond C, Barker DJ, et al. Size at birth, maternal weight, and type 2 diabetes in South India. Diabet Med. 1998;15:220-7. Internet. Medline:9545123 doi:10.1002/(SICI)1096-9136(199803)15:3<220::AIDDIA544>3.0.CO;2-O

29 Dabelea D, Mayer-Davis EJ, Lamichhane AP, D'Agostino RB, Liese AD, Vehik KS, et al. Association of intrauterine exposure to maternal diabetes and obesity with type 2 diabetes in youth the SEARCH case-control study. Diabetes Care. 2008;31:1422-6. Medline:18375420 doi:10.2337/dc07-2417

30 Aschim EL, Grotmol T, Tretli S, Haugen TB. Is there an association between maternal weight and the risk of testicular cancer? An epidemiologic study of Norwegian data with emphasis on World War II. Int J Cancer. 2005;116:327-30. Medline:15800917 doi:10.1002/ijc.21044

31 Sanderson M, Williams MA, Daling JR, Holt VL, Malone KE, Self SG, et al. Maternal factors and breast cancer risk among young women. Paediatr Perinat Epidemiol. 1998;12:397-407. Medline:9805713 doi:10.1046/j.1365-3016.1998.00133.x

32 Hansen S, Maslova E, Strøm M, Linneberg A, Halldorsson TI, Granström C, et al. The long-term programming effect of maternal 25-hydroxyvitamin D in pregnancy on allergic airway disease and lung function in offspring after 20 to 25 years of follow-up. J Allergy Clin Immunol. 2015;136:169-76-e2. Medline:25649083 doi:10.1016/j.jaci.2014.12.1924

33 Leddy MA, Power ML, Schulkin J. The impact of maternal obesity on maternal and fetal health. Rev Obstet Gynecol. 2008;1:170-8. Medline:19173021

34 Radulescu L, Munteanu O, Popa F, Cirstoiu M. The implications and consequences of maternal obesity on fetal intrauterine growth restriction. J Med Life. 2013;6:292. Medline:24155784

35 McDonald SD, Han Z, Mulla S, Beyene J. Overweight and obesity in mothers and risk of preterm birth and low birth weight infants: systematic review and meta-analyses. BMJ. 2010;341:c3428. Medline:20647282 doi:10.1136/bmj.c3428

36 Frankel S, Elwood P, Smith GD, Sweetnam P, Yarnell J. Birthweight, body-mass index in middle age, and incident coronary heart disease. Lancet. 1996;348:1478-80. Medline:8942776 doi:10.1016/S0140-6736(96)03482-4

37 Barker DJP, Osmond C, Winter PD, Margetts B, Simmonds SJ. Weight in infancy and death from ischaemic heart disease. Lancet. 1989;334:577-80. Medline:2570282 doi:10.1016/S0140-6736(89)90710-1

38 Hult M, Tornhammar P, Ueda P, Chima C, Bonamy AKE, Ozumba B, et al. Hypertension, diabetes and overweight: Looming legacies of the biafran famine. PLoS One. 2010;5:e13582. Medline:21042579 doi:10.1371/journal.pone.0013582

39 Hales CN, Barker DJP. The thrifty phenotype hypothesis. Br Med Bull. 2001;60:5-20. Medline:11809615 doi:10.1093/ $\mathrm{bmb} / 60.1 .5$

40 Martin-Gronert MS, Ozanne SE. Mechanisms linking suboptimal early nutrition and increased risk of type 2 diabetes and obesity. J Nutr. 2010;140:662-6. Medline:20107142 doi:10.3945/jn.109.111237

41 Dabelea D, Harrod CS. Role of developmental overnutrition in pediatric obesity and type 2 diabetes. Nutr Rev. $2013 ; 71$ suppl 1:S62-7. Medline:24147926 doi:10.1111/nure.12061

42 Clausen TD, Mathiesen ER, Hansen T, Pedersen O, Jensen DM, Lauenborg J, et al. High prevalence of type 2 diabetes and pre-diabetes in adult offspring of women with gestational diabetes mellitus or type 1 diabetes the role of intrauterine hyperglycemia. Diabetes Care. 2008;31:340-6. Medline:18000174 doi:10.2337/dc07-1596

43 Gatford KL, Simmons RA, De Blasio MJ, Robinson JS, Owens JA. Review: placental programming of postnatal diabetes and impaired insulin action after IUGR. Placenta. 2010;31:S60-5. Medline:20096455 doi:10.1016/j.placenta.2009.12.015

44 Barker DJP. The developmental origins of adult disease. J Am Coll Nutr. 2004;23(Suppl):588S-95S. Medline:15640511 doi:10.1080/07315724.2004.10719428

45 McCormack VA, dos Santos Silva I, Koupil I, Leon DA, Lithell HO. Birth characteristics and adult cancer incidence: Swedish cohort of over 11,000 men and women. Int J Cancer. 2005;115:611-7. Medline:15700315 doi:10.1002/ijc.20915

46 Bergström R, Adami H-O, Möhner M, Zatonski W, Storm H, Ekbom A, et al. Increase in testicular cancer incidence in six European countries: a birth cohort phenomenon. J Natl Cancer Inst. 1996;88:727-33. Medline:8637026 doi:10.1093/ jnci/88.11.727

47 Fabricius P, Løkke A, Marott JL, Vestbo J, Lange P. Prevalence of COPD in Copenhagen. Respir Med. 2011;105:410-7. Medline:20952174 doi:10.1016/j.rmed.2010.09.019

48 Lykkegaard J, Kristensen GN. Chronic obstructive pulmonary disease in Denmark: Age-period-cohort analysis of first-time hospitalisations and deaths 1994-2012. Respir Med. 2016;114:78-83. Medline:27109815 doi:10.1016/j. rmed.2016.03.014

49 Harpsøe MC, Basit S, Bager P, Wohlfahrt J, Benn CS, Nøhr EA, et al. Maternal obesity, gestational weight gain, and risk of asthma and atopic disease in offspring: a study within the Danish National Birth Cohort. J Allergy Clin Immunol. 2013;131:1033-40. Medline:23122630 doi:10.1016/j.jaci.2012.09.008 
50 Wei Z, Zhang J, Yu X. Maternal vitamin D status and childhood asthma, wheeze and eczema: a systematic review and meta-analysis. Pediatr Allergy Immunol. 2016;27:612-9. Medline:27145360 doi:10.1111/pai.12593

51 Wang Z, Zou Z, Yang Z, Dong Y, Ma J. Association between exposure to the Chinese famine during infancy and the risk of self-reported chronic lung diseases in adulthood: a cross-sectional study. BMJ Open. 2017;7:e015476. Medline:28576899 doi:10.1136/bmjopen-2016-015476

52 Martinez FD. Early-Life Origins of Chronic Obstructive Pulmonary Disease. N Engl J Med. 2016;375:871-8. Medline:27579637 doi:10.1056/NEJMra1603287

53 Ferland-McCollough D, Fernandez-Twinn DS, Cannell IG, David H, Warner M, Vaag AA, et al. Programming of adipose tissue miR-483-3p and GDF-3 expression by maternal diet in type 2 diabetes. Cell Death Differ. 2012;19:1003-12. Medline:22223106 doi:10.1038/cdd.2011.183

54 Sandovici I, Smith NH, Nitert MD, Ackers-Johnson M, Uribe-Lewis S, Ito Y, et al. Maternal diet and aging alter the epigenetic control of a promoter-enhancer interaction at the Hnf4a gene in rat pancreatic islets. Proc Natl Acad Sci U S A. 2011;108:5449-54. Medline:21385945 doi:10.1073/pnas.1019007108

55 Smith J, Cianflone K, Biron S, Hould FS, Lebel S, Marceau S, et al. Effects of maternal surgical weight loss in mothers on intergenerational transmission of obesity. J Clin Endocrinol Metab. 2009;94:4275-83. Medline:19820018 doi:10.1210/ jc.2009-0709 